\title{
Home-based psychological nursing interventions for improvement of sleep quality and psychological health in patients with hypopharyngeal carcinoma undergoing surgical resections: a randomized trial
}

\author{
Xuelan Li, Lijun Chen, Beimei Lei, Changning Xie \\ Teaching and Research Section of Clinical Nursing, Xiangya Hospital of Central South University, Changsha, China \\ Contributions: (I) Conception and design: X Li; (II) Administrative support: None; (III) Provision of study materials or patients: L Chen; (IV) \\ Collection and assembly of data: B Lei; (V) Data analysis and interpretation: C Xie; (VI) Manuscript writing: All authors; (VII) Final approval of \\ manuscript: All authors. \\ Correspondence to: Changning Xie. Teaching and Research Section of Clinical Nursing, Xiangya Hospital of Central South University, 86 Xiangya \\ Road, Kaifu District, Changsha 410008, China. Email: xcn13875848186@163.com.
}

Background: The primary aim of this study was to determine the efficacy of a psychological nursing
intervention for improving sleep quality and alleviating anxiety and depression among hypopharyngeal
carcinoma patients undergoing surgical resections.
Methods: A total of 140 hypopharyngeal carcinoma patients undergoing surgical resections in our hospital
from January 2017 to December 2020 were included and randomly assigned to a standard nursing group or
psychological nursing group, with 70 cases per group. Sleep quality was assessed using the Pittsburgh sleep
quality index (PSQI). The severity of anxiety symptoms and depressive disorders were evaluated using Zung's
self-rating anxiety scale (SAS) and self-rating depression scale (SDS). The functional assessment of cancer
therapy-general scale (FACT-G) tool was used to evaluate the quality of life. Fear of progression (FoP) was
also scored.

Results: Participants with hypopharyngeal carcinoma in the psychological nursing group demonstrated greater improvements in the primary outcome of global sleep quality $(\mathrm{P}=0.002)$ at postintervention compared to the participants in the standard nursing group. The psychological nursing group participants showed remarkably greater improvements in the subscale scores of global sleep quality characteristics (secondary outcomes) including daytime dysfunction $(\mathrm{P}<0.001)$, subjective sleep quality $(\mathrm{P}=0.029)$, and sleep latency $(\mathrm{P}=0.006)$ at postintervention compared to those receiving standard nursing. Psychological nursing group participants had lower SAS and SDS scores compared to those in the standard nursing group $(\mathrm{P}<0.001)$. The results revealed that participants who received psychological nursing intervention exhibited higher scores of all FACT-G subscales and lower scores of FoP compared to participants receiving standard nursing intervention (both $\mathrm{P}<0.001$ ). The global sleep quality scores shared positive associations with scores of SAS, SDS, and FoP ( $r=0.518, r=0.572$, and $r=0.395$, respectively, $\mathrm{P}<0.001)$.

Conclusions: These data provide evidence that home-based psychological nursing intervention could improve sleep quality and alleviate anxiety and depression among hypopharyngeal carcinoma patients undergoing surgical resections.

Trial Registration: Chinese Clinical Trial Registry ChiCTR2100051463.

Keywords: Hypopharyngeal carcinoma; psychological nursing intervention; sleep quality; anxiety; depression

Submitted Oct 05, 2021. Accepted for publication Nov 15, 2021.

doi: 10.21037/apm-21-3029

View this article at: https://dx.doi.org/10.21037/apm-21-3029 


\section{Introduction}

Hypopharyngeal carcinoma is a malignant tumor that occurs in the nasopharyngeal mucosa, which is adjacent to many important organs, blood vessels, and nerves (1). Anatomically speaking, the lateral pharynx, posterior pharyngeal wall, piriform sinus, and retrocricoid area leading to the entrance of the esophagus are involved in the structure of the hypopharynx (2). The specific pathogenesis of hypopharyngeal carcinoma still remains unclear. It involves interactions in multiple genes, living environment, lifestyle, and risk factors including viral infection, low selenium intake, and excessive nickel intake (3). Clinically, it is not easy to treat patients with hypopharyngeal carcinoma, due to various elements such as the advanced stage at which they frequently present, poor physical condition, severe complications, and excessive drinking habits (4). It has been reported that about $60-85 \%$ patients with hypopharyngeal carcinoma are diagnosed as III-IV stage, resulting in poor prognosis regardless of active multidisciplinary treatment (5). Although hypopharyngeal carcinoma only accounts for about $3-5 \%$ of all head and neck cancers, it is one of the diseases with poor prognosis among such cancers, which has a reported 5 -year overall survival rate of about 30-35\% (6). In addition, nearly $50 \%$ of patients with hypopharyngeal carcinoma are likely to relapse within the first year after treatment, and are usually diagnosed with distant metastasis, which eventually leads to a $64 \%$ chance of death (7). Laryngectomy is the mainstay of treatment for hypopharyngeal carcinoma (8). After total laryngectomy, the patients lose their vocal function and they fail to communicate with others satisfactorily. In addition, when the patients receiving total laryngectomy underwent tracheostomy, they keep ventilation from the trachea to the lung, leading to frequent dryness. Moreover, the patients lose their self-confidence due to direct discharge of sputum and secretions from the ostomy mouth. Patients with laryngeal preservation are better than those with total laryngectomy in these aspects, but they have poor swallowing function. All these postoperative complications in surgery lead to psychological changes involving anxiety, depression, and worry, poor treatment compliance, and decreased quality of life (9). Therefore, postoperative nursing is very important for patients undergoing surgical resections for hypopharyngeal carcinoma, such as keeping indoor air fresh, oral care of patients, increasing atomization inhalation, providing guidance for patients to eat, and observing the color and nature of patients' secretions. Sleep problems among cancer survivors are common in the first five-year post-treatment, and sleep difficulty may cluster with pain, fatigue, physical limitations, depression, and distress symptoms in early survivorship (10). The emotional toll of cancer, often captured as mood disturbance, anxiety, depression, and intrusive thoughts, contributes to sleep disruptions within the first five years of survivorship as well. Psychological nursing intervention refers to any action or activity taken by nurses to improve cancer patients' mental health, which is a new nursing management mode that focuses on the psychological processes of patients. It can reduce the physiological and psychological stress of patients, and accelerate their recovery (11). Accumulated evidence has shown the effectiveness of psychological nursing interventions among patients with cancer $(12,13)$; however, current psychological nursing intervention studies for patients with hypopharyngeal carcinoma only focus on psychoeducation implemented directed toward the patients, ignore social support and the involvement of family members. Patients with hypopharyngeal carcinoma undergoing surgery may experience social stigmatization. Familial problems, especially spousal problems, affected patient's life. It is important for patients to share their feelings that they experience during a disease process such as fear and unhappiness with their spouses or family members. Their spouses should also consider disease as a familial problem, understand cancer-related difficulties experienced by women, be willing to share their feelings, and display a supportive attitude toward women's coping attempts. Spouses' or family members' failure to fulfill them has an adverse effect on coping and psychological well-being of cancer patients. In this study, a home-based psychological nursing intervention model was applied to hypopharyngeal carcinoma patients receiving surgery, and its influence on sleep quality and emotional health was explored. We present the following article in accordance with the CONSORT reporting checklist (available at https://dx.doi.org/10.21037/apm-21-3029).

\section{Methods}

\section{Participants and recruitment criteria}

Patients who had been initially diagnosed with hypopharyngeal squamous cell carcinoma and were to receive surgical resections in our hospital were recruited to the study. To be eligible, patients with hypopharyngeal carcinoma must have had a predicted survival of 
$>3$ months after surgical resections, been aged 18-65 years, Karnofsky performance scores (KPS) $\geq 70$, and simple intelligent mental state examination scale (MMSE) score of $\geq 27$. Patients were excluded for the following reasons: presence of distant metastasis; confirmed diagnosis of sleep apnea; heart, lung, liver, and kidney dysfunction; endocrine and metabolic diseases, such as hypothyroidism, hyperthyroidism, and metabolic syndrome; illiteracy; impaired verbal communication; failure to complete the questionnaire due to various reasons; psychiatric disorders; pregnancy and lactation; experiencing or experienced other psychological nursing experiments. Eligible patients were assigned to either a standard nursing or psychological nursing group by simple randomization, with 70 cases per group. This is a two-parallel study and allocation ratio is 1:1. The study was approved by the Ethics Committee of the Xiangya Hospital of Central South University (approval number: 202108146), and all participants provided written informed consent. All procedures performed in this study involving human participants were in accordance with the Declaration of Helsinki (as revised in 2013).

\section{Nursing protocols}

Participants in the standard nursing group were provided with relevant information about hypopharyngeal carcinoma, such as pathogenesis, objective of surgical resection, and precautions during the perioperative period. In this period, the participants were given standard nursing care for better compliance and cooperation to surgery. Participants in the psychological nursing group underwent 5 weeks of homebased psychological nursing interventions. During the first week, health education was performed by nursing staff with substantial clinical experience and good communication skills to explain the concept, symptoms, and risk factors of hypopharyngeal carcinoma. Psychological evaluation was performed on each participant, and it was ensured that their family members also understood their condition. The nursing staff educated the patients and their family members about the dietary, medication, and basic nursing care according to therapeutic regimes and clinical manifestations, and ensured that family members grasped the importance of their participation in the process of psychological nursing to help improve the participants' mood. In the second week, the participants were explained the objective of surgical resection and precautions during the perioperative period. According to psychological status of the participant, they were given psychogenic relaxation therapy for self-control, self-training, and confidence regarding surgery with encouragement and support from their family members. At the third week, family-oriented, ordinary, and life-oriented communication was established to improve nurse-patient relations, create a family-oriented language atmosphere, and improve patient communication enthusiasm, nursing, and treatment compliance. During the fourth week, a session was conducted to clearly explain to the patients and their family members the matters needing attention after surgery and how to exercise for physical recovery, and to broaden their understanding of hypopharyngeal carcinoma. At the fifth week, $1 \mathrm{~h}$ conversations about hypopharyngeal carcinoma and precautions after surgery were facilitated between participants and their family members. All participants underwent surgical resections.

\section{Pittsburgh sleep quality index (PSQI)}

The Chinese version of the PSQI was adapted to measure sleep quality over a 1-month interval. The Chinese PSQI is the same as the traditional PSQI reported by Buysse et al. (14) and includes 19 self-rated questions covering 7 different components: subjective sleep quality, sleep latency, sleep duration, habitual sleep efficiency, sleep disturbances, sleep medication use, and daytime dysfunction. The global sleep quality score was the primary outcome, and the subscale scores of global sleep quality characteristics were secondary outcomes. Component scores that ranged from 0 to 3 were summed to provide a global sleep quality score ranging from 0 to 21 . Higher scores indicated poorer sleep quality, and participants with a score $>7$ were regarded as those having poor sleep quality.

\section{Zung's self-rating anxiety scale (SAS) and self-rating depression scale (SDS)}

The Chinese versions of the Zung's SAS and SDS were employed to evaluate the severity of anxiety symptoms and depressive disorders. The SAS and the SDS encompass both psychological and somatic symptoms. Participants were required to self-respond to the questions based on their experiences and feelings. Both of them include 20 items and were scored on a 4-point Likert scale (1 represents never or very infrequently, 2 represents some of the time, 3 represents good part of the time, and 4 represents most or all of the time), and can intuitively reflect respondents' subjective feelings for nearly 1 week. The raw scores ranged from 20 to 80 and was transformed to a standardized total 
score ranging from 25 to 100 . Higher scores indicated more obvious anxiety and depression. A score $\geq 50$ represented a reasonable cut-off point to identify cases of anxiety and depression. Previous studies have shown that both the SAS and SDS have good internal consistency with a Cronbach's $\alpha$ coefficient of 0.827 and 0.898 , respectively (15).

\section{Functional assessment of cancer therapy-general scale (FACT-G)}

The Chinese version of the FACT-G was translated from version 3 of FACT-G. This is a 29-item instrument with each item scored on $1-5$ response categories ( 0 represents "not at all", 1 represents "a little bit", 2 represents "Somewhat", 3 represents "quite a bit", and 4 represents "very much". The items reflect cancer treatment-related impacts on quality of life. The FACT-G has 5 subscales, measuring physical, social/family, emotional, and function.

\section{Fear of progression (FoP) questionnaire short form}

The FoP questionnaire short form is a 12 -item instrument with 2 fields (physical health and social family) and its Chinese version (with Cronbach's $\alpha$ coefficient of 0.88 ) was used to assess FoP (16). This instrument used a Likert 5-point scale with end anchors of 1 representing "never" and 5 representing "very often", finally attaining a resulting sum score ranging from 12 to 60 . Higher scores indicated greater degree of FoP, and a score $\geq 34$ reflected a dysfunctional level of FoP.

\section{Statistical analysis}

The data obtained were statistically processed using GraphPad Prism8 (GraphPad Software, La Jolla, CA, USA). Quantitative data were shown as the mean \pm standard deviation, with Student's $t$-test used for 2-group comparison. Count data were expressed as percentages (\%), with chi-squared tests used for 2-group comparison. A P value of $<0.05$ (2 tailed) was regarded as evidence of significant difference.

\section{Results}

\section{Demographic information of study participants}

Participants reported withdrawing largely for personal reasons, illness, and treatment-related issues, not because of dissatisfaction with the psychological nursing intervention. Finally, a total of 140 patients who had been initially diagnosed with hypopharyngeal carcinoma and underwent surgical resections in our hospital from January 2017 to December 2020 were included in the study according to our inclusion and exclusion criteria (participant flow seen in Figure 1). Table 1 shows the baseline characteristics for the 140 participants who were consented and eligible for the study, and the baseline data were separated into the two study arms: the standard nursing and psychological nursing groups. No significant differences were noted with regard to age, gender, tobacco consumption, alcohol drinking, areca chewing, education level, marital status, income, residence, oral hygiene, clinical stage, tumor type, laryngeal function preservation, and neck dissection between the standard nursing and psychological nursing groups. All participants completed all questionnaires before and after interventions. A trained nurse conducted the survey and guided the patients to fill the questionnaires with unified guidelines. After doing a good job of explanation, the patient shall fill in the form item by item, and the questionnaire shall be collected on site.

\section{The incidence of postoperative complications for patients with hypopharyngeal carcinoma}

Considering the incidence of postoperative complications, there were 3 cases of incision infection, 1 case of lung infection, 3 cases of lymphocystis, and 4 cases of skin paralysis in the standard nursing group, whereas there were 2 cases of incision infection, 2 cases of lymphocystis, and 3 cases of skin paralysis in the psychological nursing group. No significant difference was found concerning the incidence rates of postoperative complications between the standard nursing and psychological nursing groups $(\mathrm{P}>0.05$, Table 2).

\section{Home-based psychological nursing improved postoperative sleep quality of patients with bypopharyngeal carcinoma}

The sleep quality of the participants with hypopharyngeal carcinoma before and after intervention was evaluated by patient-reported sleep quality variables from PSQI. As shown in Table 3, participants with hypopharyngeal carcinoma in the psychological nursing group demonstrated greater improvements in the primary outcome of global sleep quality $(\mathrm{P}=0.002)$ at postintervention compared to those in the standard nursing group. Additionally, 


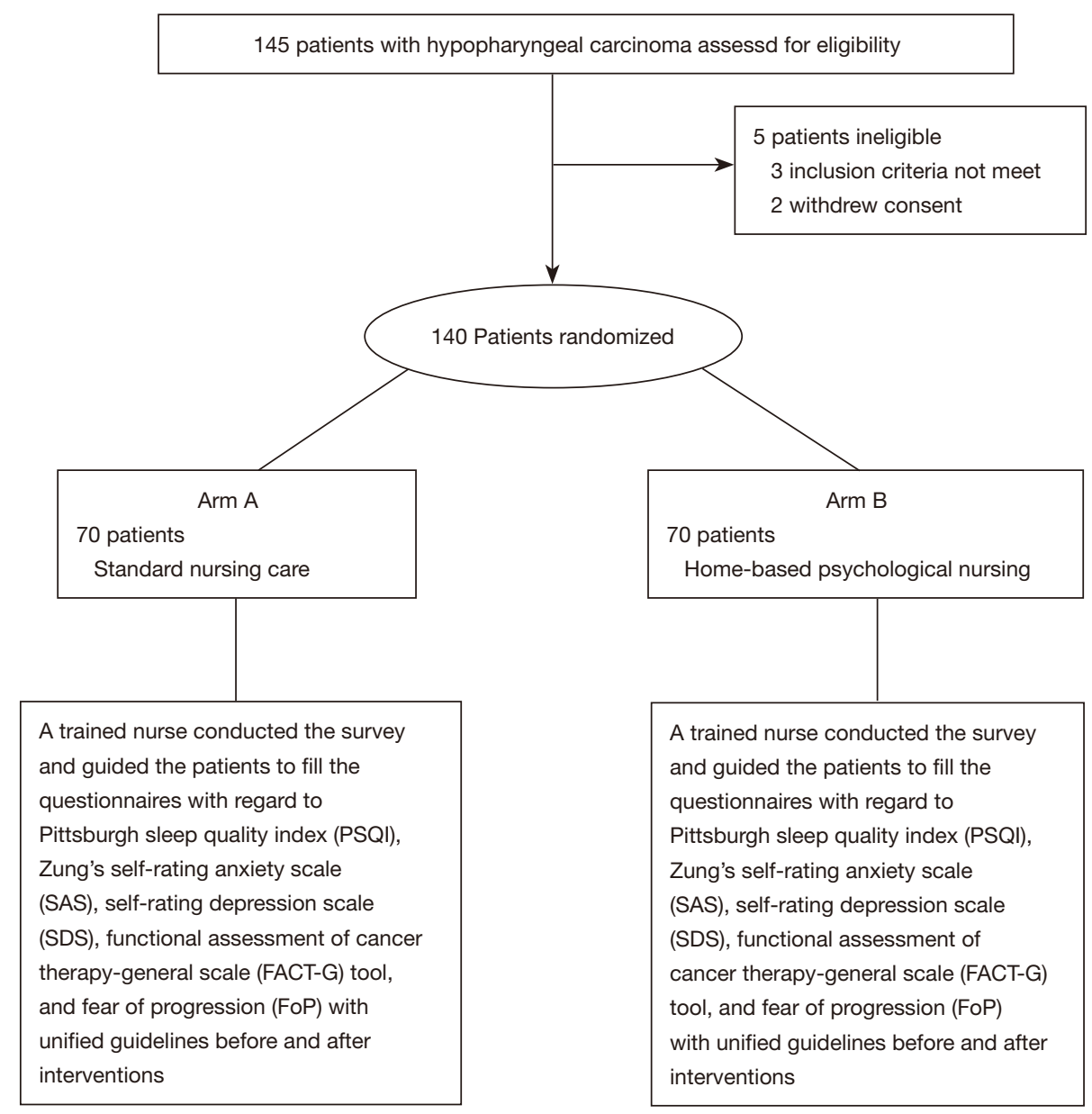

Figure 1 The participant flow of the study.

participants receiving psychological nursing showed remarkably greater improvements in the subscale scores of global sleep quality characteristics (secondary outcomes) including daytime dysfunction $(\mathrm{P}<0.001)$, subjective sleep quality $(\mathrm{P}=0.029)$, and sleep latency $(\mathrm{P}=0.006)$ at postintervention compared to those receiving standard nursing. According to a score $>7$ indicating poor sleep quality, there were 49 participants with poor sleep quality in the standard nursing group and 36 participants with poor sleep quality in the psychological nursing group after intervention, showing a statistical difference $(70.00 \% v s$. $51.43 \%, \mathrm{P}=0.037)$. These data suggest that home-based psychological nursing intervention could improve the sleep quality of patients with hypopharyngeal carcinoma after surgical resections.
Home-based psychological nursing alleviated depressive and anxious symptoms of patients with hypopharyngeal carcinoma after surgery

Next, we evaluated depression and anxiety of patients with hypopharyngeal carcinoma before and after intervention by using Zung's SAS and SDS questionnaires. As listed in Table 4, the SAS and SDS scores of the participants with hypopharyngeal carcinoma in both groups were decreased after intervention; participants in the psychological nursing group had lower SAS and SDS scores compared to those in the standard nursing group $(\mathrm{P}<0.001)$. These data suggest that psychological nursing intervention could effectively alleviate depression and anxiety of patients with hypopharyngeal carcinoma. 
Table 1 Baseline characteristics of the 140 participants

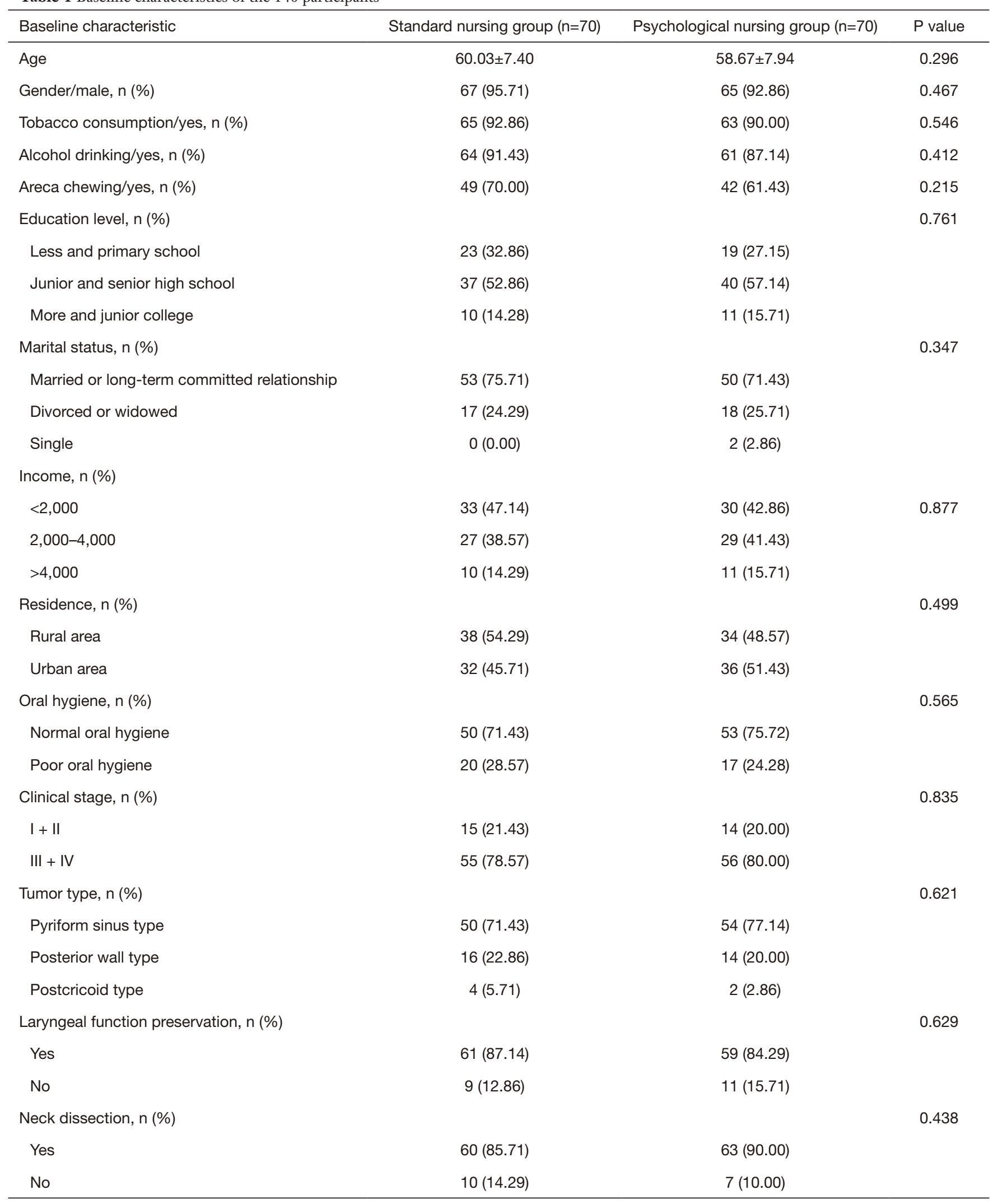


Table 2 Incidence rates of postoperative complications in the standard nursing and psychological nursing groups

\begin{tabular}{lcccc}
\hline Group & Incision infection & Lung infection & Lymphocystis & Skin paralysis \\
\hline Standard nursing group $(\mathrm{n}=70)$ & 3 & 1 & 3 & Total \\
Psychological nursing group $(\mathrm{n}=70)$ & 2 & 0 & 2 & 4 \\
P value & & & $3(15.71 \%)$ & $7(10.00 \%)$ \\
\hline
\end{tabular}

Table 3 Patient-reported sleep quality variables from PSQI before and after intervention in the standard nursing and psychological nursing groups

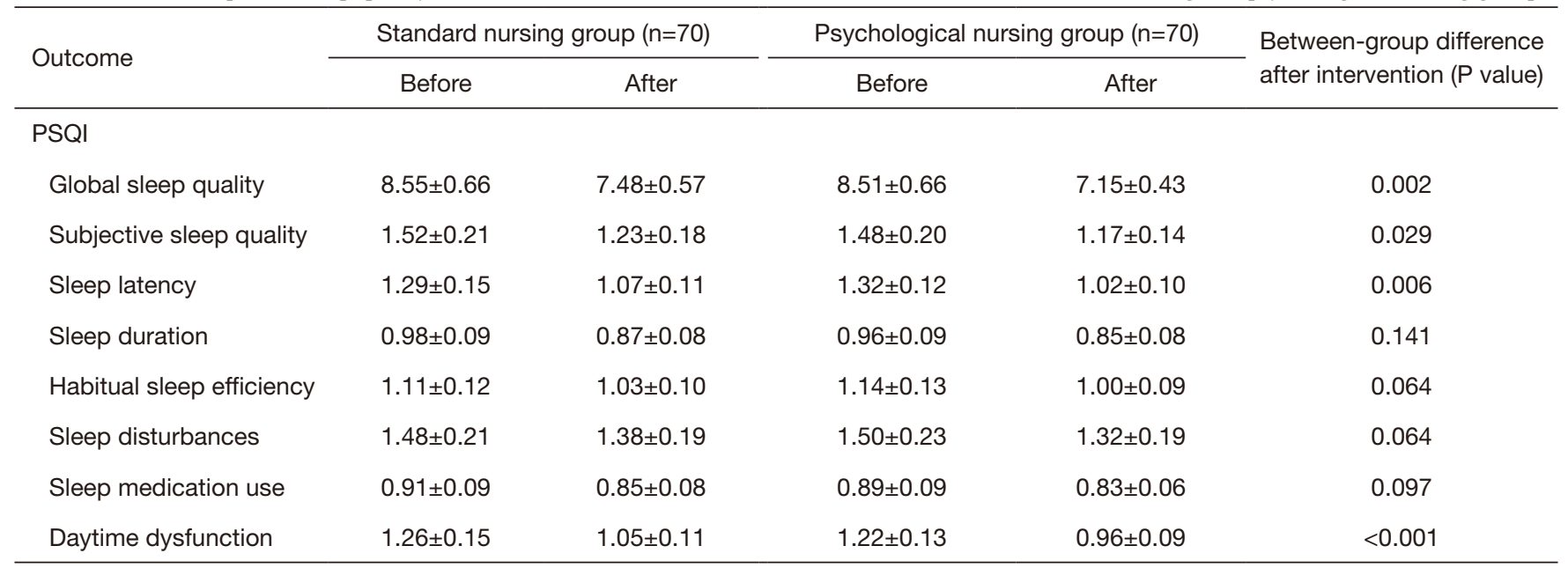

PSQI, Pittsburg sleep quality index.

Table 4 The depression and anxiety of patients with hypopharyngeal carcinoma before and after intervention by using Zung's SAS and SDS questionnaires in the standard nursing and psychological nursing groups

\begin{tabular}{lccccccc}
\hline \multirow{2}{*}{ Outcome } & \multicolumn{2}{c}{ Standard nursing group $(\mathrm{n}=70)$} & & \multicolumn{2}{c}{ Psychological nursing group $(\mathrm{n}=70)$} & \multicolumn{2}{c}{$\begin{array}{c}\text { Between-group difference after } \\
\text { intervention }(\mathrm{P})\end{array}$} \\
\cline { 2 - 3 } & Before & After & & Before & After & $<0.001$ \\
SAS & $55.27 \pm 9.59$ & $48.55 \pm 7.85$ & & $54.57 \pm 8.78$ & $33.49 \pm 5.74$ & $<0.001$ \\
SDS & $45.13 \pm 6.26$ & $39.97 \pm 5.44$ & & $44.99 \pm 7.16$ & $36.27 \pm 5.62$ & $<$ \\
\hline
\end{tabular}

SAS, self-rated anxiety scale; SDS, self-rated depression scale.

\section{Home-based psychological nursing increased quality of life of patients with hypopharyngeal carcinoma after surgery}

Quality of life is increasingly recognized as an important outcome of cancer treatment. The FACT-G tool was used to evaluate the impact of psychological nursing intervention on the quality of life of patients with hypopharyngeal carcinoma after surgery. The results revealed that the participants receiving psychological nursing intervention exhibited higher scores of all FACT-G subscales, physical, social/family, emotional, and function when compared to those receiving standard nursing intervention $(\mathrm{P}<0.001$,
Table 5). Fear about cancer recurrence or progression is a common challenge experienced by individuals living with and beyond cancer and is endorsed as the highest unmet psychosocial need amongst survivors. The FoP scores of patients with hypopharyngeal carcinoma were $33.83 \pm 3.86$ and $34.03 \pm 4.73$ in the standard nursing and psychological nursing groups before intervention, respectively. The FoP scores of patients were $34.78 \pm 3.31$ and $29.87 \pm 2.01$ in the standard nursing and psychological nursing groups after intervention, respectively. It was found that the patients in the psychological nursing group displayed lower scores of FoP than those in the standard nursing group $(\mathrm{P}<0.001)$. 
Table 5 The FACT-G scores of patients with hypopharyngeal carcinoma after intervention in the standard nursing and psychological nursing groups

\begin{tabular}{lccc}
\hline Outcome & Standard nursing group $(\mathrm{n}=70)$ & Psychological nursing group $(\mathrm{n}=70)$ & $\mathrm{P}$ value \\
\hline Physical & $20.26 \pm 2.93$ & $26.58 \pm 3.24$ & $<0.001$ \\
Emotional & $19.12 \pm 4.04$ & $25.45 \pm 3.10$ & $<0.001$ \\
Social/family & $17.59 \pm 3.19$ & $23.33 \pm 3.78$ & $<0.001$ \\
Function & $18.44 \pm 3.56$ & $22.08 \pm 3.26$ & $<0.001$ \\
\hline
\end{tabular}

FACT-G, functional assessment of cancer therapy-general scale.
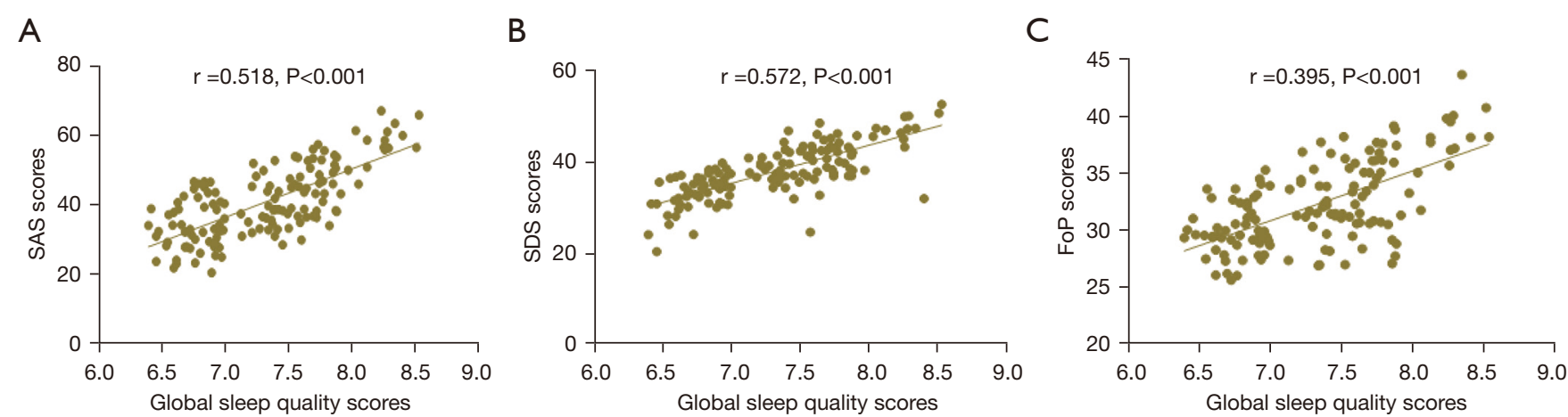

Figure 2 The relationships between sleep quality, depression and anxiety, and fear of cancer progression of patients with hypopharyngeal carcinoma after surgery. SAS, self-rating anxiety scale; SDS, self-rating depression scale; FoP, fear of progression.

\section{Relationship between sleep quality and psychological bealth in patients with bypopharyngeal carcinoma after surgery}

In the following analyses, we investigated the relationships between sleep quality, depression and anxiety, and fear of cancer progression of patients with hypopharyngeal carcinoma after surgery. The global sleep quality scores were found to share positive associations with scores of SAS, SDS, and FoP $(\mathrm{r}=0.518, \mathrm{r}=0.572, \mathrm{r}=0.395, \mathrm{P}<0.001$, Figure $2 A-2 C)$. These results showed that poorer sleep quality reflects a more severely negative emotional state.

\section{Discussion}

Hypopharyngeal carcinoma is considered a painless and asymptomatic tumor, which is rare among all head and neck cancers, accounting for approximately $3-5 \%$. Clinically, it is usually diagnosed at an advanced stage due to the asymptomatic nature of these tumors, leading to a high risk of death (17). In planning the treatment of these patients, 3 major obstacles are faced, including complicated condition due to advanced stage disease, controversial optimal scheme of treatment, and insufficient knowledge of this cancer which might be due to its low incidence (7). Recently, it has been demonstrated that surgery could be associated with better survival outcomes in patients with hypopharyngeal carcinoma; however, the optimal treatment requires further research (18). To date, a multitude of research has reported that psychosocial nursing interventions in cancer patients are effective in addressing treatment and physical symptoms, such as quality of life (19), helplessness, and depression (20). However, there have been studies conducted on psychosocial nursing in patients with hypopharyngeal carcinoma. In this study, 140 hypopharyngeal carcinoma patients undergoing surgery were randomly assigned to standard nursing and psychological nursing groups, with 70 cases in each group. We recruited hypopharyngeal carcinoma patients who received surgery in order to explore the improvement of psychosocial nursing intervention on postoperative complications, psychological emotions, and sleep quality.

Obviously, postoperative complications are related to all kinds of surgeries, such as those for cardiovascular disease, orthopedic surgery, and various cancers. Due to the special location of the hypopharynx, which is adjacent 
to many important structures, nerves, blood vessels, there are certain complications associated with surgery for hypopharyngeal carcinoma (21). According to the data of postoperative complications we observed, there was no significant difference in the incidence of postoperative complications, involving incision infection, lung infection, lymphocystis, and skin paralysis, between the psychological nursing group and standard nursing group. However, a similar research on nasopharyngeal carcinoma patients undergoing radiotherapy demonstrated that the incidence of adverse reactions, including oral mucosal reaction, skin reaction, nausea and vomiting, and leukocyte decline, were significantly lower in patients treated with highquality nursing intervention than those who received routine nursing intervention (22). This contrasts with our outcomes, which might be due to the different treatments and location of disease. At present, it is generally believed that psychological factors play an important role in the process of carcinogenesis, that is, cancer patients are more prone to unconscious or conscious psychological symptoms such as stress, depression, and anxiety than benign tumor patients (12). This study evaluated the depression and anxiety of patients with hypopharyngeal carcinoma before and after intervention, and compared it to those who received standard nursing invention, which revealed that those who received psychological nursing invention had lower SAS and SDS scores. In addition, to further explore the contribution of psychological nursing invention to the experience of sleep symptoms, a series of sleep assessments were performed, which indicated, in terms of PSQI scale, that the psychological nursing group achieved remarkable improvements in the primary outcome of global sleep quality and in the subscale scores of global sleep quality characteristics. The findings suggested that depression, anxiety, nervousness, and sleep disorders were alleviated by the psychological nursing invention, which aligns with previous studies that have indicated psychological nursing invention helps cancer patients to develop their fighting spirit (20), and had a significantly large positive effect on physical and psychological symptoms, such as pain, dizziness, sleep disturbance, worrying, depression, and irritability $(23,24)$.

Overall, the quality of life in hypopharyngeal carcinoma patients received psychological nursing intervention was improved, which was reflected in the higher scores of all FACT-G subscales, physical, social/family, emotional, and function. It was similar to another study wherein $\mathrm{Li}$ et al. noted that high-quality nursing intervention greatly improved the quality of life in patients with nasopharyngeal carcinoma undergoing radiotherapy, encompassing a variety of scores such as general health score, physical functioning score, bodily pain score, and mental health score, and so on (22). Similarly, Hershman et al. revealed that nursing care in breast cancer patients improved their quality of life and prolonged their survival time (25), and a study of patients with hepatocellular carcinoma who underwent surgery indicated that comprehensive education and care alleviated anxiety and depression, and improved quality of life (26). With the improvement of cancer survival rate, fear of cancer recurrence has become a prominent clinical problem. It is a common and painful process, leading to negative impacts on patients' quality of life and medical burden (27). Considering the high recurrence rate of hypopharyngeal carcinoma, an analysis of FoP scores was conducted, and it was demonstrated the participants in the psychological nursing group displayed lower scores of FoP than those in the standard nursing group. Similar to our findings, Hall et al. (28) pointed out mind-body interventions were effective in reducing fear of cancer recurrence.

It has been reported that physical symptoms (e.g., fatigue and pain), poor general health, increased awareness of symptoms, insufficient preparation for lingering symptoms, the lack of social support, fear of recurrences, negative automatic thoughts, and poor quality of life were risk factors of distress, depression, and anxiety for most cancer survivors. The World Health Organization defines homebased nursing as any form of care given to ill people in their homes and with the help of their family members, including physical, psychosocial, palliative and spiritual activities. This psychological nursing intervention is based on family nursing, with subjective initiative of the patient's family members to support patients. Therefore each patient will be given corresponding psychological nursing according to their situations, which helps the patient establish the confidence to overcome the disease and deal with the possible adverse reactions during the perioperative period, so as to reduce the patient's fear, help the patient establish the confidence to overcome the disease and eliminate the negative emotion, improve patients' treatment compliance and cooperation. ensured that their family members also understood their condition. The nursing staff educated the patients and their family members about the dietary, medication, and basic nursing care according to therapeutic regimes and clinical manifestations, and ensured that family members grasped the importance of their participation in the process of psychological nursing to help improve the 
participants' mood. In the second week, the participants were explained the objective of surgical resection and precautions during the perioperative period. According to psychological status of the participant, they were given psychogenic relaxation therapy for self-control, self-training, and confidence regarding surgery with encouragement and support from their family members. In addition to that, when given home-based psychological nursing, the patients can listen to music, read books, and talk their bad emotions under a warm, comfortable and pleasant hospitalization environment.

In general, this study demonstrated home-based psychological nursing intervention could improve sleep quality and alleviate anxiety and depression among hypopharyngeal carcinoma patients undergoing surgical resections. Sleep quality was closely associated with emotional state and reasonable and effective psychological intervention was of great benefit to patients with hypopharyngeal carcinoma undergoing surgery. Homebased psychological nursing interventions help the patients to relieve negative emotional symptoms, such as helpless, depression, anxiety, and fear of cancer recurrence, prevent sleep disorder, and increase quality of life. However, this study had several limitations. The study was conducted with a relatively small patient sample, which prevents the research results from being extended to other patients, treatment environments, and different disease stages. Due to the high risk of recurrence rate of hypopharyngeal carcinoma, 5 weeks of psychological nursing invention might be insufficient to the patients, and further followingup studies should be conducted in the near future.

\section{Acknowledgments}

Funding: This study received funding from Application of Accelerated Rehabilitation Surgical Nursing in Patients with Hypopharyngeal Cancer, Number: Hunan Caijiao Zhi (2018) No. 33.

\section{Footnote}

Reporting Checklist: The authors have completed the CONSORT reporting checklist. Available at https://dx.doi. org/10.21037/apm-21-3029

Trial Protocol: Available at https://dx.doi.org/10.21037/apm21-3029
Data Sharing Statement: Available at https://dx.doi. org/10.21037/apm-21-3029

Conflicts of Interest: All authors have completed the ICMJE uniform disclosure form (available at https://dx.doi. org/10.21037/apm-21-3029). The authors have no conflicts of interest to declare.

Ethical Statement: The authors are accountable for all aspects of the work in ensuring that questions related to the accuracy or integrity of any part of the work are appropriately investigated and resolved. The study was approved by the Ethics Committee of the Xiangya Hospital of Central South University (approval number: 202108146), and all participants provided written informed consent. All procedures performed in this study involving human participants were in accordance with the Declaration of Helsinki (as revised in 2013).

Open Access Statement: This is an Open Access article distributed in accordance with the Creative Commons Attribution-NonCommercial-NoDerivs 4.0 International License (CC BY-NC-ND 4.0), which permits the noncommercial replication and distribution of the article with the strict proviso that no changes or edits are made and the original work is properly cited (including links to both the formal publication through the relevant DOI and the license). See: https://creativecommons.org/licenses/by-nc-nd/4.0/.

\section{References}

1. Qian J, Zhu J. The clinical curative strategies of hypopharyngeal carcinoma. Lin Chung Er Bi Yan Hou Tou Jing Wai Ke Za Zhi 2017;31:1701-4.

2. Ball M, Hossain M, Padalia D. Anatomy, Airway. StatPearls, Ed., Treasure Island (FL), 2021.

3. Li Y, Lu T, Hu G. Gene sequencing and expression of Raf1 in lymphatic metastasis of hypopharyngeal carcinoma. Cancer Biomark 2020;28:181-91.

4. Garneau JC, Bakst RL, Miles BA. Hypopharyngeal cancer: A state of the art review. Oral Oncol 2018;86:244-50.

5. Sewnaik A, Hoorweg JJ, Knegt PP, et al. Treatment of hypopharyngeal carcinoma: analysis of nationwide study in the Netherlands over a 10-year period. Clin Otolaryngol 2005;30:52-7.

6. Newman JR, Connolly TM, Illing EA, et al. Survival trends in hypopharyngeal cancer: a population-based 
review. Laryngoscope 2015;125:624-9.

7. Hall SF, Groome PA, Irish J, et al. The natural history of patients with squamous cell carcinoma of the hypopharynx. Laryngoscope 2008;118:1362-71.

8. Chan JY, Wei WI. Current management strategy of hypopharyngeal carcinoma. Auris Nasus Larynx 2013;40:2-6.

9. Lu J, Zeng X, Liao J, et al. Effectiveness of an Intervention to Promote Self-Efficacy on Quality of Life of Patients with Nasopharyngeal Carcinoma of the Zhuang Tribe Minority in Guangxi, China: A Prospective Study. Med Sci Monit 2017;23:4077-86.

10. Strollo SE, Fallon EA, Gapstur SM, et al. Cancer-related problems, sleep quality, and sleep disturbance among longterm cancer survivors at 9-years post diagnosis. Sleep Med 2020;65:177-85.

11. Tuominen L, Stolt M, Meretoja R, et al. Effectiveness of nursing interventions among patients with cancer: An overview of systematic reviews. J Clin Nurs 2019;28:2401-19.

12. Wu L, Zou Y. Psychological nursing intervention reduces psychological distress in patients with thyroid cancer: A randomized clinical trial protocol. Medicine (Baltimore) 2020;99:e22346.

13. Schleisman A, Mahon E. Creative Play: A Nursing Intervention for Children and Adults With Cancer Clin J Oncol Nurs 2018;22:137-40.

14. Buysse DJ, Reynolds CF 3rd, Monk TH, et al. The Pittsburgh Sleep Quality Index: a new instrument for psychiatric practice and research. Psychiatry Res 1989;28:193-213.

15. Li X, Yu H, Yang W, et al. Depression and Anxiety Among Quarantined People, Community Workers, Medical Staff, and General Population in the Early Stage of COVID-19 Epidemic. Front Psychol 2021;12:638985.

16. Hinz A, Mehnert A, Ernst J, et al. Fear of progression in patients 6 months after cancer rehabilitation-a- validation study of the fear of progression questionnaire FoP-Q-12. Support Care Cancer 2015;23:1579-87.

17. Tsai YT, Chen WC, Chien CY, et al. Treatment patterns and survival outcomes of advanced hypopharyngeal squamous cell carcinoma. World J Surg Oncol 2020;18:82.

18. Mu L, Liu X, Liu X, et al. Loss of CDH1 promotes the metastasis of hypopharyngeal squamous cell carcinoma through the STAT3-MMP-9 signaling pathway. Transl Cancer Res 2019;8:1476-85.

19. Bakitas M, Lyons KD, Hegel MT, et al. Effects of a palliative care intervention on clinical outcomes in patients with advanced cancer: the Project ENABLE II randomized controlled trial. JAMA 2009;302:741-9.

20. Rodin G, Lloyd N, Katz M, et al. The treatment of depression in cancer patients: a systematic review. Support Care Cancer 2007;15:123-36.

21. Tokashiki K, Okamoto I, Okada T, et al. Postoperative Complications of Jejunal and Skin Valve Reconstruction in Free Reconstructive Surgery for Hypopharyngeal Carcinoma. Anticancer Res 2021;41:4033-8.

22. Li C, Duan J. Effect of high-quality nursing intervention on psychological emotion, life quality and nursing satisfaction of patients with nasopharyngeal carcinoma undergoing radiotherapy. Am J Transl Res 2021;13:4928-38.

23. Tsvitman I, Castel OC, Dagan E. The association between perceived patient-centered care and symptoms experienced by patients undergoing anti-cancer treatment. Support Care Cancer 2021;29:6279-87.

24. Park S, Sato Y, Takita Y, et al. Mindfulness-Based Cognitive Therapy for Psychological Distress, Fear of Cancer Recurrence, Fatigue, Spiritual Well-Being, and Quality of Life in Patients With Breast Cancer-A Randomized Controlled Trial. J Pain Symptom Manage 2020;60:381-9.

25. Hershman DL, Ganz PA. Quality of Care, Including Survivorship Care Plans. Adv Exp Med Biol 2015;862:255-69.

26. Wang J, Yan C, Fu A. A randomized clinical trial of comprehensive education and care program compared to basic care for reducing anxiety and depression and improving quality of life and survival in patients with hepatocellular carcinoma who underwent surgery. Medicine (Baltimore) 2019;98:e17552.

27. Sharpe L, Curran L, Butow P, et al. Fear of cancer recurrence and death anxiety. Psychooncology 2018;27:2559-65.

28. Hall DL, Luberto CM, Philpotts LL, et al. Mindbody interventions for fear of cancer recurrence: A systematic review and meta-analysis. Psychooncology 2018;27:2546-58.

(English Language Editor: J. Jones)

Cite this article as: Li X, Chen L, Lei B, Xie C. Home-based psychological nursing interventions for improvement of sleep quality and psychological health in patients with hypopharyngeal carcinoma undergoing surgical resections: a randomized trial. Ann Palliat Med 2021;10(12):12347-12357. doi: 10.21037/apm-213029 